\title{
Predicting the properties of first cycle inbreds and second cycle hybrids extractable from two, three and four parent crosses
}

\author{
H. S. Pooni and \\ J. L. Jinks
}

Department of Genetics, University of Birmingham, P.O. Box 363, Birmingham B15 2TT. U.K.

\begin{abstract}
Standard biometrical genetical models of Mather and Jinks (1982) when made applicable to the means and variances of the 55 early generations produced by crossing four parents and six $F_{1}$ 's in all possible combinations provide estimates of genetic parameters that can be used to predict the distributive properties of the first cycle inbreds and second cycle hybrids which could be extracted from any of these generations. Thus we can predict the inbreeding and outbreeding potentials of each generation in the early stages of a breeding programme and formulate the best breeding strategy for harnessing the full genetic potential of the breeding material and choosing the best end product. The 55 generations provide reliable estimates of the predictors and therefore should be used whenever possible. Simpler experiments consisting of the basic generations of the six single crosses, however, are sufficient for obtaining estimates of the predictors of the inbreds and can be used to predict their properties when information about the second cycle hybrids is not required or the remaining generations are not available. Replacement of the $F_{2}$, backcross, three way cross and double cross generations with their randomly mated progenies is expected to improve the accuracy of predictions in the presence of a linkage disequilibrium. However, the gains made may not be justified against the costs of the additional breeding and the delay in making the predictions.

Extensive experimental testing of the theory of these procedures must await the completion of the current field experiments. In the meantime the limited tests conducted on the material extracted from the Nicotiana rustica varieties $V_{1}, V_{2}, V_{5}$ and $V_{12}$ have confirmed the familiar conclusions that the $V_{1} \times V_{5}$ and $V_{2} \times V_{12}$ differ in their inbreeding and outcrossing potentials.
\end{abstract}

\section{INTRODUCTION}

During the last few years we have devised methods for predicting the inbreeding and outbreeding potentials of a cross between two pure breeding varieties (Jinks and Perkins, 1972; Jinks and Pooni, 1976; Pooni and Jinks, 1978; 1979; Toledo, Pooni and Jinks, 1984a, $b$; Pooni, Jinks and Toledo, 1985). These methods have proved to be highly reliable for making predictions on a wide range of materials (Coombs,1980; Tapsell and Thomas, 1983; Thomas and Tapsell, 1983) and equally so in simple and complex genetical situations. The information required for making these predictions is easily obtainable from the early generations of the cross.

In the present paper we extend these procedures to the first cycle inbred lines and second cycle hybrids which can be extracted from backcross, threeway cross and double cross populations produced by intercrossing of two, three and four parents respectively. We shall develop theoretical models which account for up to four alleles at any number of loci and utilize parameters that can be estimated from the early generations. We shall illustrate the theory by analysing experiments involving four inbred varieties of Nicotiana rustica raised in 1978 and 1983.

\section{GENERATIONS}

Pairwise crossing and selfing of each of four parental varieties and six possible $F_{1}$ 's yield 55 unique families which can be produced in two seasons. These include 28 families which correspond with 
the $P_{1}, P_{2}, F_{1}, F_{2}, B_{1}$ and $B_{2}$ generations of each of the six single crosses. Genetic and environmental variability of the means and variances of the six generations in each set, therefore, can be partitioned according to Mather and Jinks (1982) and predictions made following Jinks and Pooni (1976) and Toledo et al. (1984a,b). Such a model, however, assumes that each set of basic generations is independent of all others. It explicitly ignores the allelic relationships that exist between the different sets of basic generations.

The remaining 27 generations are multiple crosses between three or four parental varieties. Twelve of these generations are three way crosses of the type $\left(P_{1} \times P_{2}\right) \times P_{3}$, another twelve are double crosses with one parent common to the two $F_{1}$ 's that produce the double cross of the type $\left(P_{1} \times\right.$ $\left.P_{2}\right) \times\left(P_{1} \times P_{3}\right)$ and three are double crosses that involve all four parents of the type $\left(P_{1} \times P_{2}\right) \times$ $\left(P_{3} \times P_{4}\right)$ but different pairs of $F_{1}$ 's. Segregation at any locus in these generations can involve up to four alleles simultaneously.

\section{THEORETICAL MODELS}

Of the $K$ loci that control genetic variability of a metrical trait let there be $k(k \leqq K)$ loci for which the four parents have different alleles fixed among them. For some of these loci $\left(k^{\mathrm{Il}}\right)$ the four parents will incorporate two alleles, for others $\left(k^{\text {III }}\right)$ three alleles and for the rest $\left(k^{\text {IV }}\right)$ four alleles such that $k^{\mathrm{II}}+k^{\mathrm{III}}+k^{\mathrm{IV}}=k$. The remaining $(K-k)$ loci will by definition have the same allele fixed in each parent and therefore they will not contribute to the genetic variability between parents and their crosses.

\section{3i. Loci with two alleles}

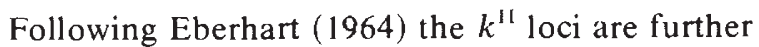
divided into various groups depending upon the frequencies of alleles at each locus and their distribution among the parents. For example, the frequencies of $A: a$ alleles at locus $A / a$ can be $\frac{3}{4}: \frac{1}{4}$ or $\frac{1}{4}: \frac{3}{4}$ or $\frac{1}{2}: \frac{1}{2}$. Further, there are eight phases in which $A A$ or $a a$ can be present amongst the four parents when their frequencies are $\frac{3}{4}: \frac{1}{4}$ or $1: \frac{3}{4}$ and another six phases when they are equally frequent. However, only seven of these combinations are unique and the remaining seven are their mirror images as shown in table 1. Because everyone of

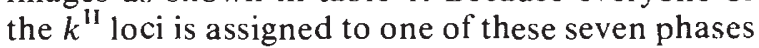
we require 14 parameters to account for the additive and dominance effects of these seven groups of loci. We define these parameters as

$$
d_{1}=\sum_{i=1}^{k_{1}^{\prime \prime}} d_{i} ; \quad d_{2}=\sum_{i=1}^{k_{2}^{\prime \prime}} d_{i} \ldots d_{7}=\sum_{i=1}^{k ! n} d_{i}
$$

and

$$
h_{1}=\sum_{i=1}^{k_{1}^{\prime \prime}} h_{i} ; \quad h_{2}=\sum_{i=1}^{k_{2}^{\prime \prime}} h_{i} \ldots h_{7}=\sum_{i=1}^{k_{7}^{\prime \prime}} h_{i}
$$

where $d_{1}$ to $d_{7}$ and $h_{1}$ to $h_{7}$ are the additive and dominance parameters and $d_{i}\left(+d_{a}\right.$ for $A A$ and $-d_{a}$ for $\left.a a\right)$ and $h_{i}\left(h_{a}\right.$ for $\left.A a\right)$ are the additive and dominance effects of individual loci. Also $k_{1}^{1 \mathrm{I}}+$ $k_{2}^{\mathrm{II}}+\ldots k_{7}^{\mathrm{II}}=k^{\mathrm{II}}$.

Contributions of the seven additive parameters to the means of the four parents are given in table 2. Expectations of the remaining generations can be obtained by substituting these coefficients for each of the seven groups of loci into the general formulae given by Mather and Jinks (1982) for the $F_{1}, F_{2}$ and backcross generations of a single cross. For most of the generations these expectations are the same as those given by Eberhart (1964). Further, each parameter takes an internal sign and the constant $m^{\prime}$ is the mean of an $F_{x x}$ population where allelic frequencies are equal for each of the $k^{\text {II }}$ loci.

The corresponding expectations of the genetic variances for each generation are also obtained by using the respective formulae of Mather and Jinks (1982) for the $F_{2}$ and backcross variances. Here, we require 21 parameters, i.e., one $D$, one $H$ and one $F$ for each of the seven groups of loci and we denote them as $V d_{1}$ to $V d_{7}, V h_{1}$ to $V h_{7}$ and $W d h_{1}$ to $W d h_{7}$, respectively.

Table 1 Alternative phases of " $A$ " and " $a$ " alleles on a single locus amongst the four inbred parents

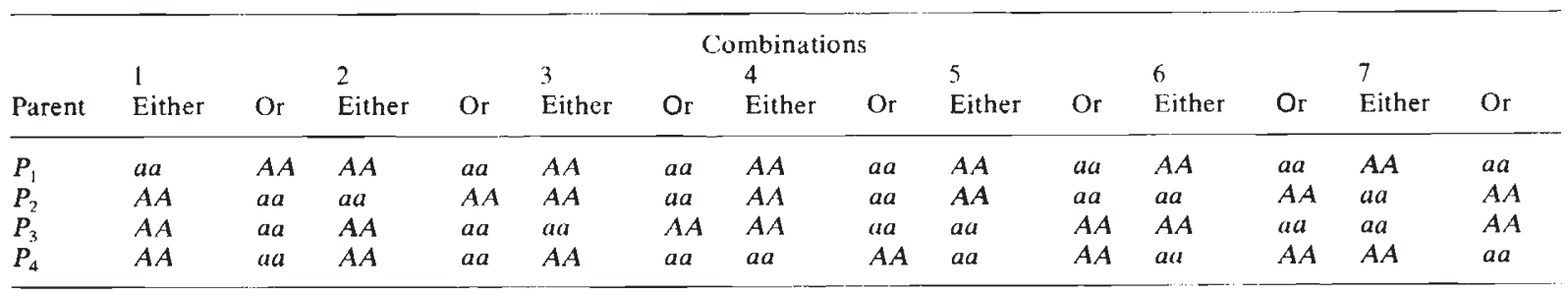


Table 2 Additive genetic contributions of the seven groups of loci to the means of the parental varieties

\begin{tabular}{lrrrrrrrr}
\hline & \multicolumn{7}{c}{ Parameters } \\
Parent & $m^{\prime}$ & $d_{1}$ & $d_{2}$ & $d_{3}$ & $d_{4}$ & $d_{5}$ & $d_{6}$ & $d_{7}$ \\
\hline$P_{1}$ & 1 & -1 & 1 & 1 & 1 & 1 & 1 & 1 \\
$P_{2}$ & 1 & 1 & -1 & 1 & 1 & 1 & -1 & -1 \\
$P_{3}$ & 1 & 1 & 1 & -1 & 1 & -1 & 1 & -1 \\
$P_{4}$ & 1 & 1 & 1 & 1 & -1 & -1 & -1 & 1 \\
\hline
\end{tabular}

\section{3ii. Loci with three or four alleles}

There are 12 and 16 phases in which three and four alleles can be distributed amongst four parents. Again there are only seven unique phases and we can assign additional parameters to account for their specific effects. However, all of these parameters have strong aliases with the parameters that are already defined in section $3 i$. The alternative is to define the effects of three and four alleles in such a way that their effects are either confounded with the parameters defined previously or are accounted for as epistasis or linkage disequilibrium or both.

Differences between the additive effects of four alleles, $A_{1}, A_{2}, A_{3}$ and $A_{4}$ can be equated with the four parents being $A A B B, A A b b, a a B B$ and $a a b b$ on a digenic model. However, monogenic and digenic crosses produce different segregation ratios and the above equation is therefore true only if there is no recombination between the $A / a$ and $B / b$ loci. The dominance relationships of four alleles, for example, $A_{1}>A_{2}>A_{3}>A_{4}$ or $A_{1}=$ $A_{2}>A_{3}>A_{4}$, etc. can also be equated on the digenic model where alleles $A$ and $B$ are dominant to alleles $a$ and $b$ or alleles $A$ and $b$ are dominant to alleles $a$ and $B$, respectively. The triallelic situation is simply represented as an imbalance between the four genotypes (given earlier) where one of the genotypes is missing and the other represented twice.

On this model we require four parameters, $2 d$ 's and $2 h$ 's, to assign the effects of alleles at each locus and to confound their effects with existing parameters. For example, if $P_{1}, P_{2}, P_{3}$ and $P_{4}$ are $A_{1} A_{1}, A_{2} A_{2}, A_{3} A_{3}$ and $A_{4} A_{4}$ then their effects are confounded with parameters $d_{5}, h_{5}, d_{6}, h_{6}, d_{7}$ and $h_{7}$, respectively. If, however, they are $A_{2} A_{2}, A_{3} A_{3}$, $A_{1} A_{1}$ and $A_{1} A_{1}$ then their effects are confounded with parameters $d_{4}, h_{4}, d_{2}, h_{2}, d_{5}$ and $h_{5}$.

\section{3iii. Non-allelic interaction and linkage disequilibrium}

We can extend the additive/dominance model to accommodate the digenic interactions. For example, we can parameterise the epistatic effects of each of the seven groups of loci by allocating seven additive $\times$ additive, 14 additive $\times$ dominance and seven dominance $\times$ dominance interaction parameters. We shall therefore require 28 additional parameters to account for the three kinds of epistatic effects which can arise between the genes of various groups of loci. Obviously the number of parameters required are very large and they will be aliased intricately. Therefore, it will be sensible to confound these parameters with one another or with parameters that are least correlated.

Linkage dis-equilibrium, in the absence of nonallelic interactions, does not affect the expectations of the generation means. It does, however, inflate or deflate the genetic variances of the generations depending upon the net balance between the coupling and repulsion linkages. Its overall effects on the estimates of components of variances, nevertheless, are expected to be minimal because multiple crosses allow more chances for the alternate phases of linkage to be represented amongst the segregants.

\section{ESTIMATION OF PARAMETERS}

The number of parameters in the model described in section 3 is too large to be estimated from the generations we intend to use for making predictions. However, these parameters can be used to obtain the expectations of the statistics whose estimates we require for predicting the properties of first cycle inbreds and second cycle hybrids. Further, because the estimates of $m,[d]$ and $[h]$ and $D, H$ and $F$ components (see Mather and Jinks, (1982), for definitions) will normally be available from the generation means and generation variances of the basic generations of single crosses, we shall attempt to establish theoretical relationships between these and the new parameters.

The relationships between the components of means $m,[d]$ and $[h]$ of each single cross and the $m^{\prime}$ and additive and dominance parameters specified in section 3 are described in table 3 . These relationships are also valid for the three way and doublecross families. The alternative expectations of these generations and the six sets of basic generations from single crosses are given in tables 4 and 5. Parameters $m,[d]$ and $[h]$ of each cross are subscripted by the numbers assigned to the parents of that cross. For example $m_{13},[d]_{13}$ and $[h]_{13}$ are the parameters for the generations extracted from the $P_{1} \times P_{3}$ cross. Efficient estimates of each of 
Table 3 Relationships between $m,[d]$ and $[h]$ components of the single crosses and the $m^{\prime}$ and fourteen $d$ and $h$ components of the seven groups of loci described in table 1

\begin{tabular}{llll}
\hline Cross $m$ & {$[d]$} & {$[h]$} \\
\hline $1 \times 2+$ & $m^{\prime}+d_{3}+d_{4}+d_{5}^{*}$ & $-d_{1}+d_{2}+d_{6}+d_{7}$ & $h_{1}+h_{2}+h_{6}+h_{7}$ \\
$1 \times 3$ & $m^{\prime}+d_{2}+d_{4}+d_{6}$ & $-d_{1}+d_{3}+d_{5}+d_{7}$ & $h_{1}+h_{3}+h_{5}+h_{7}$ \\
$1 \times 4$ & $m^{\prime}+d_{2}+d_{3}+d_{7}$ & $-d_{1}+d_{4}+d_{5}+d_{6}$ & $h_{1}+h_{4}+h_{5}+h_{6}$ \\
$2 \times 3$ & $m^{\prime}+d_{1}+d_{4}-d_{7}$ & $-d_{2}+d_{3}+d_{5}-d_{6}$ & $h_{2}+h_{3}+h_{5}+h_{6}$ \\
$2 \times 4$ & $m^{\prime}+d_{1}+d_{3}-d_{6}$ & $-d_{2}+d_{4}+d_{5}-d_{7}$ & $h_{2}+h_{4}+h_{5}+h_{7}$ \\
$3 \times 4$ & $m^{\prime}+d_{1}+d_{2}-d_{5}$ & $-d_{3}+d_{4}+d_{6}-d_{7}$ & $h_{3}+h_{4}+h_{6}+h_{7}$ \\
\hline
\end{tabular}

* Definitions of $m^{\prime}, d_{1}$ and $h_{1}$ etc. are given in section 3 .

$+1=P_{1}, 2=P_{2}, 3=P_{3}, 4=P_{4}$.

these parameters can be obtained by weighted least squares analysis of the means of the 55 generations.

The corresponding relationships between the components of variances $D, H$ and $F$ of each cross and the $V d_{1}, V h_{1}$ and $W d h_{1}$ etc. are shown in table 6 . These relationships however remain valid only for (1) the single crosses and their descendant generations that are derived from them through selfing and backcrossing and (2) for the inbred populations extracted from each of the 45 segregating generations. A combined model which retains the dominance $\left(V h_{1}\right.$ to $\left.V h_{7}\right)$ and covariance $\left(W d h_{1}\right.$ to $\left.W d h_{7}\right)$ parameters and substitutes the additive $\left(V d_{1}\right.$ to $\left.V d_{7}\right)$ parameters for those of single crosses ( $D_{12}, D_{13}, \ldots, D_{34}$ etc), however, is sufficient to explain the genetic variability amongst the 55 variances and permits a weighted least squares estimation of the parameters. Expectations of various generations on this model are given in table 7 .

\section{NOTATION AND PARAMETERS FOR PREDICTIONS}

In general we shall use symbols $F_{\infty}, B_{1} S_{\infty \times}, B_{2} S_{\infty}$, $T c_{1} S_{\infty}$ and $D c_{1} S_{\infty}$ to represent random inbred lines extracted from the $F_{2}, B_{1}, B_{2}$, three way and double cross generations; $\bar{F}_{\infty}, \bar{B}_{1} S_{\infty}, \bar{B}_{2} S_{\infty}, \bar{T} c_{1} S_{\infty}$ and $\bar{D} c_{1} S_{\infty}$ to represent their overall means and $\sigma_{b F \infty}^{2}$, $\sigma_{b B 1 S \alpha ;}^{2}, \sigma_{b B 2 S(x)}^{2}, \sigma_{b T_{1} \mid S_{\infty},}^{2}$ and $\sigma_{b D_{1} \mid S_{\infty}}^{2}$ to represent their genetic variances which are, of course, all between families components $\left(\sigma_{b}^{2}\right)$. Further, each symbol will be labelled by the source of origin of the inbreds to distinguish them from one another. For example $F_{\infty}\left(P_{1} \times P_{2}\right)$ and $F_{\infty}\left(P_{3} \times P_{4}\right)$ represent inbred lines that are extracted from $P_{1} \times P_{2}$ and $P_{3} \times P_{4}$ crosses respectively.

Table 4 Genetical expectations of the six single crosses, $6 F_{2}$ 's and 12 first backcrosses in terms of $m$ 's $[d]$ 's and [ $h$ ]'s when they are unique for each of the six crosses

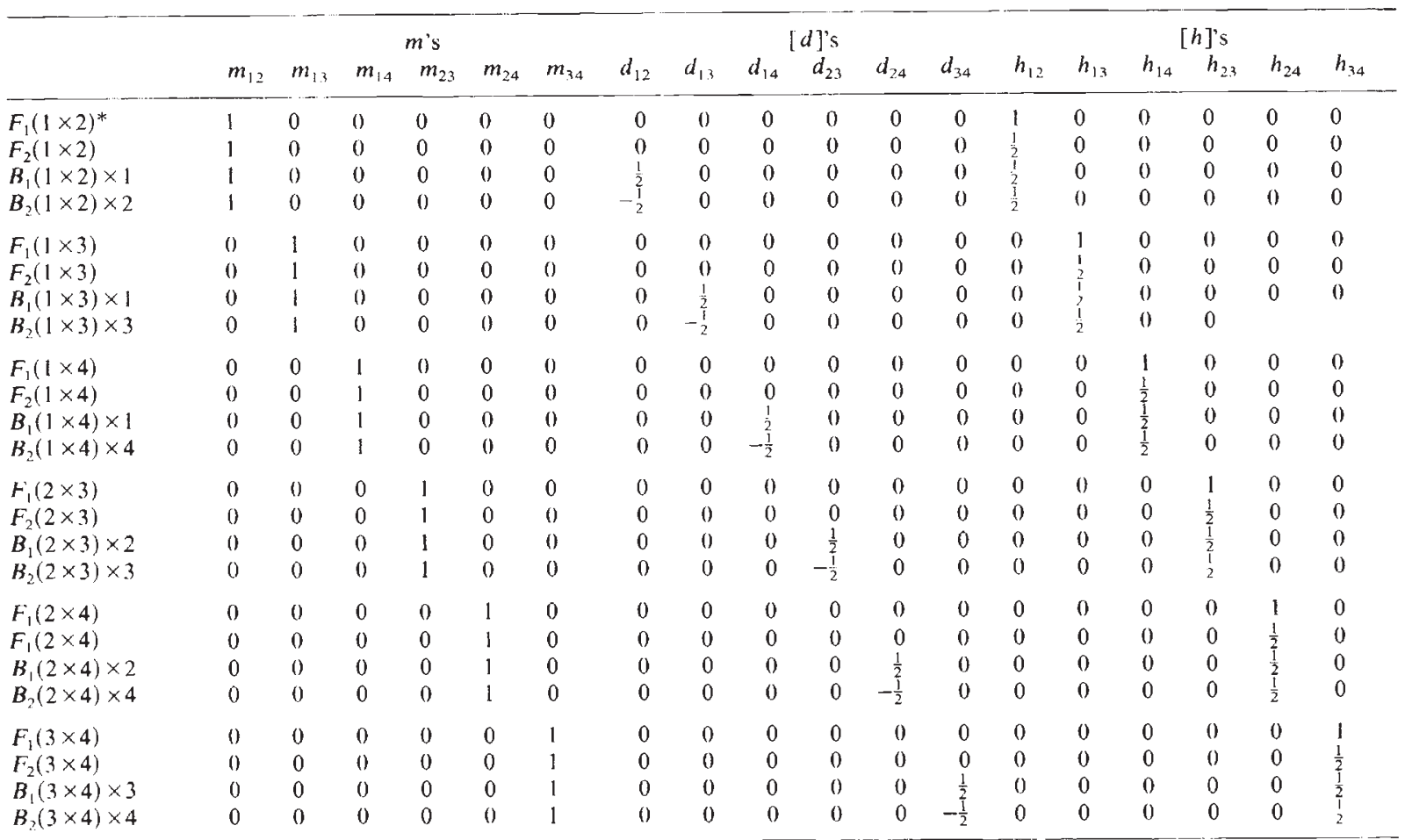

* See table 3. 
Table 5 Contributions of $m$ 's and [ $h$ ]'s to the means of the three way and double crosses when these parameters are unique for each of the six crosses which can be produced by the intercrossing of four parents

\begin{tabular}{lllllllllllll}
\hline & \multicolumn{4}{c}{ The " $m$ " parameters } & \multicolumn{4}{c}{ The "[ $h]$ " parameters } \\
Generation & $m_{12}$ & $m_{13}$ & $m_{14}$ & $m_{23}$ & $m_{24}$ & $m_{34}$ & $h_{12}$ & $h_{13}$ & $h_{14}$ & $h_{23}$ & $h_{24}$ & $h_{34}$ \\
\hline
\end{tabular}

(a) Three way crosses

$$
\begin{aligned}
& (1 \times 2) \times 3 \ddagger \\
& (1 \times 2) \times 4 \\
& (1 \times 3) \times 2 \\
& (1 \times 3) \times 4 \\
& (1 \times 4) \times 2 \\
& (1 \times 4) \times 3 \\
& (2 \times 3) \times 1 \\
& (2 \times 3) \times 4 \\
& (2 \times 4) \times 1 \\
& (2 \times 4) \times 3 \\
& (3 \times 4) \times 1 \\
& (3 \times 4) \times 2
\end{aligned}
$$

\begin{tabular}{|c|c|c|c|c|c|c|c|c|c|c|c|}
\hline$(1 \times 2) \times(1 \times 3)$ & $\frac{1}{2}$ & $\frac{1}{2}$ & 0 & 0 & 0 & 0 & $\frac{1}{4}$ & $\frac{1}{4}$ & 0 & $\frac{1}{4}$ & 0 \\
\hline$(1 \times 2) \times(1 \times 4)$ & $\frac{1}{2}$ & 0 & $\frac{1}{2}$ & 0 & 0 & 0 & $\frac{1}{4}$ & 0 & $\frac{1}{4}$ & 0 & $\frac{1}{4}$ \\
\hline$(1 \times 2) \times(2 \times 3)$ & $\frac{1}{2}$ & 0 & 0 & $\frac{1}{2}$ & 0 & 0 & $\frac{1}{4}$ & $\frac{1}{4}$ & 0 & $\frac{1}{4}$ & 0 \\
\hline$(1 \times 2) \times(2 \times 4)$ & $\frac{1}{2}$ & 0 & 0 & 0 & $\frac{1}{2}$ & 0 & $\frac{1}{4}$ & 0 & $\frac{1}{4}$ & 0 & $\frac{1}{4}$ \\
\hline$(1 \times 3) \times(1 \times 4)$ & 0 & $\frac{1}{2}$ & $\frac{1}{2}$ & 0 & 0 & 0 & 0 & $\frac{1}{4}$ & $\frac{1}{4}$ & 0 & 0 \\
\hline$(1 \times 3) \times(2 \times 3)$ & 0 & $\frac{1}{2}$ & 0 & $\frac{1}{2}$ & 0 & 0 & $\frac{1}{4}$ & $\frac{1}{4}$ & 0 & $\frac{1}{4}$ & 0 \\
\hline$(1 \times 3) \times(3 \times 4)$ & 0 & $\frac{1}{2}$ & 0 & 0 & 0 & $\frac{1}{2}$ & 0 & $\frac{1}{4}$ & $\frac{1}{4}$ & 0 & 0 \\
\hline$(1 \times 4) \times(2 \times 4)$ & 0 & 0 & $\frac{1}{2}$ & 0 & $\frac{1}{2}$ & 0 & $\frac{1}{4}$ & 0 & $\frac{1}{4}$ & 0 & $\frac{1}{4}$ \\
\hline$(1 \times 4) \times(3 \times 4)$ & 0 & 0 & $\frac{1}{2}$ & 0 & 0 & $\frac{1}{2}$ & 0 & $\frac{1}{4}$ & $\frac{1}{4}$ & 0 & 0 \\
\hline$(2 \times 3) \times(2 \times 4)$ & 0 & 0 & 0 & $\frac{1}{2}$ & $\frac{1}{2}$ & 0 & 0 & 0 & 0 & $\frac{1}{4}$ & $\frac{1}{4}$ \\
\hline$(2 \times 3) \times(3 \times 4)$ & 0 & 0 & 0 & $\frac{1}{2}$ & 0 & $\frac{1}{2}$ & 0 & 0 & 0 & $\frac{1}{4}$ & $\frac{1}{4}$ \\
\hline$(2 \times 4) \times(3 \times 4)$ & 0 & 0 & 0 & 0 & $\frac{1}{2}$ & $\frac{1}{2}$ & 0 & 0 & 0 & $\frac{1}{4}$ & $\frac{1}{4}$ \\
\hline
\end{tabular}

\begin{tabular}{|c|c|c|c|c|c|c|c|c|c|c|c|c|}
\hline$(1 \times 2) \times(3 \times 4)$ & 0 & $\frac{1}{4}$ & $\frac{1}{4}$ & $\frac{1}{4}$ & $\frac{1}{4}$ & 0 & 0 & $\frac{1}{4}$ & $\frac{1}{4}$ & $\frac{1}{4}$ & $\frac{1}{4}$ & 0 \\
\hline$(1 \times 3) \times(2 \times 4)$ & $\frac{1}{4}$ & 0 & $\frac{1}{4}$ & $\frac{1}{4}$ & 0 & $\frac{1}{4}$ & $\frac{1}{4}$ & 0 & $\frac{1}{4}$ & $\frac{1}{4}$ & 0 & $\frac{1}{4}$ \\
\hline$(1 \times 4) \times(2 \times 3)$ & $\frac{1}{4}$ & $\frac{1}{4}$ & 0 & 0 & $\frac{1}{4}$ & $\frac{1}{4}$ & $\frac{1}{4}$ & $\frac{1}{4}$ & 0 & 0 & $\frac{1}{4}$ & $\frac{1}{4}$ \\
\hline
\end{tabular}

$\begin{array}{ll}0 & \frac{1}{2} \\ 0 & 0 \\ \frac{1}{2} & 0 \\ 0 & 0 \\ \frac{1}{2} & 0 \\ 0 & \frac{1}{2} \\ \frac{1}{2} & \frac{1}{2} \\ 0 & 0 \\ \frac{1}{2} & 0 \\ 0 & 0 \\ 0 & \frac{1}{2} \\ 0 & 0\end{array}$

$\begin{array}{ll}0 & \frac{1}{2} \\ \frac{1}{2} & 0 \\ 0 & \frac{1}{2} \\ \frac{1}{2} & 0 \\ 0 & 0 \\ 0 & 0 \\ 0 & 0 \\ 0 & 0 \\ \frac{1}{2} & 0 \\ 0 & \frac{1}{2} \\ \frac{1}{2} & 0 \\ 0 & \frac{1}{2}\end{array}$

$\begin{array}{ll}0 & 0 \\ \frac{1}{2} & 0 \\ 0 & 0 \\ 0 & \frac{1}{2} \\ \frac{1}{2} & 0 \\ 0 & \frac{1}{2} \\ 0 & 0 \\ \frac{1}{2} & \frac{1}{2} \\ 0 & 0 \\ 0 & \frac{1}{2} \\ 0 & 0 \\ \frac{1}{2} & 0\end{array}$

$\begin{array}{ll}0 & \frac{1}{2} \\ 0 & 0 \\ \frac{1}{2} & 0 \\ 0 & 0 \\ \frac{1}{2} & 0 \\ 0 & \frac{1}{2} \\ \frac{1}{2} & \frac{1}{2} \\ 0 & 0 \\ \frac{1}{2} & 0 \\ 0 & 0 \\ 0 & \frac{1}{2} \\ 0 & 0\end{array}$

$\begin{array}{cccc}0 & \frac{1}{2} & 0 & 0 \\ \frac{1}{2} & 0 & \frac{1}{2} & 0 \\ 0 & \frac{1}{2} & 0 & 0 \\ \frac{1}{2} & 0 & 0 & \frac{1}{2} \\ 0 & 0 & \frac{1}{2} & 0 \\ 0 & 0 & 0 & \frac{1}{2} \\ 0 & 0 & 0 & 0 \\ 0 & 0 & \frac{1}{2} & \frac{1}{2} \\ \frac{1}{2} & 0 & 0 & 0 \\ 0 & \frac{1}{2} & 0 & \frac{1}{2} \\ \frac{1}{2} & 0 & 0 & 0 \\ 0 & \frac{1}{2} & \frac{1}{2} & 0\end{array}$

(b) Double crosses with common parent

(c) Double crosses

The corresponding symbols that will represent the second cycle hybrids, their mean and genetic variance are $S C H, \overline{S C H}$ and $\sigma_{b S C H}^{2}$ respectively. Again each of these will be labelled according to the source of origin.

\section{5i. Random inbred lines from single crosses}

We have described on numerous occasions the theory and practice of predicting the properties of random samples of recombinant inbred lines which can be extracted from single crosses by the methods of single seed descent, pedigree inbreeding and dihaploidy (see Jinks and Pooni, 1976; 1981; 1984; Pooni and Jinks 1978; Pooni, Jinks and Cornish, 1977). To make these predictions we require estimates of the overall mean $\left(\bar{F}_{\infty}\right)$ of the $F_{\infty}$ generation of the cross. For a simple case where an additive genetic, dominance and additive environmental model is adequate, the expected overall mean of $F_{\infty}$ is $m$ and it is equal to the average performance of the two parents. For example $\bar{F}_{\infty}$ for the crosses $P_{1} \times P_{2}$ and $P_{3} \times P_{4}$ are equal to $\frac{1}{2}\left(\bar{P}_{1}+\bar{P}_{2}\right)$ and $\frac{1}{2}\left(\bar{P}_{3}+\bar{P}_{4}\right)$ respectively. More precise 
Table 6 Relationships between $D, H$ and $F$ components of the single crosses and the $V d, V h$ and $W d h$ components associated with the seven groups of loci described in tahle I

\begin{tabular}{llll}
\hline Cross & $D$ & $H$ & $-F$ \\
\hdashline $1 \times 2^{\dagger}$ & $V d_{1}^{*}+V d_{2}+V d_{6}$ & $V h_{1}+V h_{2}+V h_{6}$, & $W d h_{1}-W d h_{2}$ \\
& $+V d_{7}$ & $+V h_{7}$ & $-W d h_{6}-W d h_{7}$ \\
$1 \times 3$ & $V d_{1}+V d_{3}+V d_{5}$ & $V h_{1}+V h_{3}+V h_{5}$ & $W d h_{1}-W d h_{3}$ \\
& $+V d_{7}$ & $+V h_{7}$ & $-W d h_{5}-W d h_{7}$ \\
$1 \times 4$ & $V d_{1}+V d_{4}+V d_{5}$ & $V h_{1}+V h_{4}+V h_{5}$ & $W d h_{1}-W d h_{4}$ \\
& $+V d_{6}$ & $+V h_{6}$ & $-W d h_{5}-W d h_{6}$ \\
$2 \times 3$ & $V d_{2}+V d_{3}+V d_{5}$ & $V h_{2}+V h_{3}+V h_{5}$ & $W d h_{2}-W d h_{3}$ \\
& $+V d_{6}$ & $+V h_{6}$ & $-W d h_{5}+W d h_{6}$ \\
$2 \times 4$ & $V d_{2}+V d_{4}+V d_{5}$ & $V h_{2}+V h_{4}+V h_{5}$ & $W d h_{2}-W d h_{4}$ \\
& $+V d_{7}$ & $+V h_{7}$ & \\
& $V d_{3}+V d_{4}+V d_{6}$ & $V h_{3}+V h_{4}+V h_{6}+W d h_{7}$ & $W d h_{3}-W d h_{4}$ \\
$3 \times 4$ & $+V h_{7}$ & $-W d h_{6}+W d h_{7}$ \\
& $+V d_{7}$ & &
\end{tabular}

* $V d_{1}, V h_{1}$ and $W d h_{1}$ etc. are the additive and dominance variances and the covariance between the additive and dominance effects of individual loci within each of the seven groups of loci specified in table 1.

† See table 3 .

estimates of " $m$ ", however, can be obtained from the weighted least squares analysis of the basic generations of each cross.

Estimates of the additive genetic variance $(D)$ of the $F_{x}$ families are also readily obtained from the weighted least squares analysis of the within family variances of basic generations (see Mather and Jinks, 1982).

\section{5ii. Random inbreds from backcrosses}

The model and estimates of the components of means and variances of the single crosses can be used as such to predict the performances of inbred lines that are extractable by inbreeding the backcross generations. For instance the overall mean of these inbred lines is $m+\frac{1}{2}[d]$ when they are extracted from $B_{1}\left(F_{1} \times P_{1}\right)$ generation and $m$ $\frac{1}{2}[d]$ when they are produced from $B_{2}\left(F_{1} \times P_{2}\right)$ generation in the absence of complications like linkage disequilibrium, epistasis and genotype $\times$ environmental interactions. These means are also estimated as $\left({ }_{4}^{3} \bar{P}_{1}+\frac{1}{4} \bar{P}_{2}\right)$ and $\left(\frac{1}{4} \bar{P}_{1}+{ }_{4} \bar{P}_{2}\right)$ respectively. The genetic variance of random inbreds in either case however is equal to ${ }_{4}^{3} \mathrm{D}$, that is 75 per cent of the genetic variance of the inbred lines extracted from the $F_{2}$ of the same cross. The estimates of these parameters for the first and consecutive backerosses are given by $m+\left(1-2_{2}^{1 h}\right)[d]$ and $\left(\left(2^{b+1}-1\right) / 2^{2 b}\right) D$, where $b$ is the number of cycles of backcrossing to the same parent before selfing begins.

\section{5iii. Random inbreds from threeway and double crosses}

Estimates of parameters that are required for predicting the performances of random inbred lines extracted from any of the three way $\left(T c_{1} S_{\infty}\right)$ or double crosses $\left(D c_{1} S_{\infty}\right)$ can also be derived from the estimates of $m$ and $D$ parameters of the six single crosses. For example the overall mean $\left(T c_{1} S_{\infty}\right)$ of the inbred lines extractable from the three way cross $\left(P_{1} \times P_{2}\right) \times P_{4}$ is expected to be equal to ${ }_{2}^{1}\left(m_{14}+m_{24}\right)$ where $m_{14}$ and $m_{24}$ are the overall means of $F_{\infty}$ families of the $P_{1} \times P_{4}$ and $P_{2} \times P_{4}$ crosses. Similarly the additive genetic variance $\left(\sigma_{b T \text { TS }}^{2}\right)$ of the $\left(P_{1} \times P_{2}\right) \times P_{4}$ cross is equal to ${ }_{4}^{1} D_{12}+{ }_{2}^{1} D_{14}+{ }_{2}^{1} D_{24}$ where the three $D$ 's are the additive genetic variances of the $F_{\infty}$ families of $P_{1} \times P_{2}, P_{1} \times P_{4}$ and $P_{2} \times P_{4}$ crosses, respectively.

The expectations of $\bar{F}_{\infty}, \bar{T} c_{1} S_{\infty}$, and $\bar{D} c_{1} S_{\infty}$ for all the single, three way and double crosses are therefore the same as the expectations of their $m$ parameter and these are given in tables 4 and 5 . The expectations of $\sigma_{b F(x,}^{2}, \sigma_{b T C 1 S(x)}^{2}$ and $\sigma_{b D c \mid S: x}^{2}$ for each of these generations on the 21 parameter model (described in section 3 ) and in terms of additive genetic components $(D)$ of the six basic generations are tabulated in table 8 . These parameters can be used to obtain the proportions of inbred lines that will outperform a standard variety (St) using the formulae described by Jinks and Pooni (1976).

\section{PARAMETERS OF SECOND CYCLE HYBRIDS}

Toledo, Pooni and Jinks (1984a,b) and Pooni, Jinks and Toledo (1985) have elaborated procedures for predicting the properties of second cycle hybrids which can be produced by intercrossing a random sample of first cycle inbreds extracted from a single cross. According to them the overall mean and variance of a population of second cycle hybrids can be closely approximated to the mean and variance of a random mating population produced from the original hybrid from which the inbred lines were randomly extracted. In their case the $F_{2}$ generation was most suitable because it represented a random sample of all possible genotypes which could be produced by the random pairing of gametes from the $F_{1}$ generation. We extend the same principles to predict the hybrid potentials of the backcross, threeway cross and double cross generations when the hybrids in question are produced by the intercrossing of first cycle inbreds extracted from these 
generations by selfing. We use the parameters given in tables 4,5 and 7 to estimate the overall means and genetic variances of the second cycle hybrids as follows:

(a) for backcross generations, the overall mean of second cycle hybrids $(\overline{S C H})$ for say $\left(P_{1} \times P_{2}\right) \times$ $P_{1}$ cross is

$\overline{S C H}\left\{\left(P_{1} \times P_{2}\right) \times P_{1}\right\}=m_{12}+\frac{1}{2} d_{12}+\frac{3}{8} h_{12} ;$

(b) for three way crosses the overall mean for say $\left(P_{1} \times P_{2}\right) \times P_{3}$ is

$$
\begin{aligned}
\overline{S C H} & \left\{\left(P_{1} \times P_{2}\right) \times P_{3}\right\} \\
& =\frac{1}{2}\left(m_{13}+m_{23}\right)+\frac{1}{8}\left(h_{12}+2 h_{13}+2 h_{23}\right)
\end{aligned}
$$

(c) for double crosses with a common parent, the overall mean for say $\left(\left(P_{1} \times P_{2}\right) \times\left(P_{1} \times P_{3}\right)\right.$ is

$$
\begin{aligned}
\overline{S C H} & \left\{\left(P_{1} \times P_{2}\right) \times\left(P_{1} \times P_{3}\right)\right\} \\
& =S C H\left\{\left(P_{2} \times P_{3}\right) \times P_{1}\right\} \\
& =\frac{1}{2}\left(m_{12}+m_{13}\right)+\frac{1}{8}\left(h_{23}+2 h_{12}+2 h_{13}\right)
\end{aligned}
$$

and

(d) for double crosses the overall mean for say $\left(P_{1} \times P_{2}\right) \times\left(P_{3} \times P_{4}\right)$ is

$$
\begin{aligned}
\overline{S C H} & \left\{\left(P_{1} \times P_{2}\right) \times\left(P_{3} \times P_{4}\right)\right\} \\
= & \frac{1}{4}\left(m_{13}+m_{23}+m_{14}+m_{24}\right) \\
& +\frac{1}{8}\left(h_{12}+h_{13}+h_{14}+h_{24}+h_{34}\right)
\end{aligned}
$$

The corresponding genetic variances $\left(\sigma_{b S C H}^{2}\right)$ for the same crosses on the model given in table 7 are:

(a) $\sigma_{b S C H}^{2}\left\{\left(P_{1} \times P_{2}\right) \times P_{1}\right\}$

$$
\begin{aligned}
=\frac{3}{8} D_{12} & +\frac{15}{64}\left(V h_{1}+V h_{2}+V h_{6}+V h_{7}\right) \\
& +\frac{3}{8}\left(W d h_{1}-W d h_{2}-W d h_{6}-W d h_{7}\right) ;
\end{aligned}
$$

(b) $\sigma_{b S C H}^{2}\left\{\left(P_{1} \times P_{2}\right) \times P_{3}\right\}$

$$
\begin{aligned}
= & \frac{1}{8}\left(D_{12}+2 D_{13}+2 D_{23}\right) \\
& +\frac{15}{64}\left(V h_{1}+V h_{2}+V h_{6}+V h_{7}\right) \\
& +\frac{1}{4}\left(V h_{3}+V h_{5}\right)-\frac{3}{8}\left(W d h_{1}+W d h_{2}\right. \\
& \left.+W d h_{6}-W d h_{7}\right) ;
\end{aligned}
$$

((c) $\sigma_{h S C H}^{2}\left\{\left(P_{1} \times P_{2}\right) \times\left(P_{1} \times P_{3}\right)\right\}$

$$
\begin{aligned}
= & \frac{1}{8}\left(2 D_{12}+2 D_{13}+D_{23}\right) \\
& +\frac{15}{64}\left(V h_{2}+V h_{3}+V h_{5}+V h_{6}\right) \\
& +\frac{1}{4}\left(V h_{1}+V h_{7}\right)-\frac{3}{8}\left(W d h_{2}+W d h_{3}\right. \\
& \left.+W d h_{5}+W d h_{6}\right) ; \text { and }
\end{aligned}
$$

(d)

$$
\begin{aligned}
\sigma_{b S C H}^{2}\{ & \left.\left(P_{1} \times P_{2}\right) \times\left(P_{3} \times P_{4}\right)\right\} \\
= & \frac{1}{8}\left(D_{12}+D_{13}+D_{14}+D_{23}+D_{24}+D_{34}\right) \\
& +\frac{15}{64}\left(V h_{1}+V h_{2}+V h_{3}+V h_{4}\right) \\
& +\frac{1}{4}\left(V h_{5}+V h_{6}+V h_{7}\right) \\
& -\frac{3}{8}\left(W d h_{1}+W d h_{2}+W d h_{3}+W d h_{4}\right)
\end{aligned}
$$

Other sets of hybrids whose properties breeders may be interested in are the crosses between the $F_{\infty}$ inbreds extracted from a single cross and a recurrent parent which may or may not-be one of the two parental varieties from which the $F_{\infty}$ inbreds were extracted. The overall mean and genetic variance of such samples of hybrids usually approximate closely with those of the original cross between the $F_{1}$ from which $F_{\infty}$ inbreds were derived and the variety to which they were crossed. For example the distribution of $F_{\infty}\left(P_{1} \times P_{2}\right) \times P_{1}$ crosses approximates closely to that of $F_{1}\left(P_{1} \times\right.$ $\left.P_{2}\right) \times P_{1}$ in the absence of a linkage disequilibrium. The same is true for $F_{\infty}\left(P_{1} \times P_{2}\right) \times P_{3}$ crosses whose distribution approximates closely to that of $F_{1}\left(\mathrm{P}_{1} \times\right.$ $\left.\mathrm{P}_{2}\right) \times \mathrm{P}_{3}$.

\section{EXPERIMENTS AND RESULTS}

To test the theory and practice of predictions, we require (a) data from the 55 generations (see section 2 for description) for making predictions and (b) observations on random samples of inbred lines and second cycle hybrids for testing their validity. Experiments to generate these data are nearing completion and the results will be published in the near future. In the meantime we shall use the data that are already available to make and test the predictions.

\section{7i. Predictions}

The material for predictions consists of two sets of $P_{1}, P_{2}, F_{1}, F_{2}, B_{1}$ and $B_{2}$ generations, four three way crosses and a reciprocally produced double cross generation of $V_{1} \times V_{5}$ and $V_{2} \times V_{12}$ crosses of Nicotiana rustica. These families were raised with $20 B\left\{F_{\infty}\left(V_{1} \times V_{5}\right)\right\}$ and $20 D\left\{F_{\infty}\left(V_{2} \times V_{12}\right)\right\}$ inbred lines extracted from the $F_{2}$ generations of $V_{1} \times V_{5}$ and $V_{2} \times V_{12}$ crosses by the method of single seed descent (Perkins and Jinks, 1973; Pooni, Jinks and Jayasekara, 1978). The material was grown during the summer of 1983 as a part of a large experiment in which 20 plants were raised for each parent, 40 for each $F_{1}, 10$ for each $B$ and $D$ inbred and 25 for each of the remaining generations. Flowering time (in days from an arbitrary date) and final 


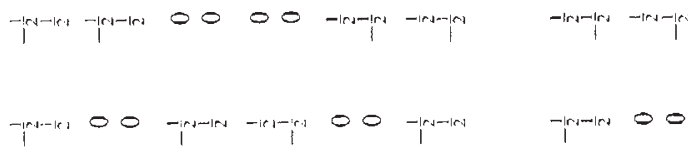

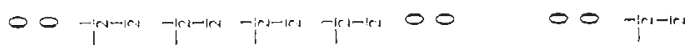

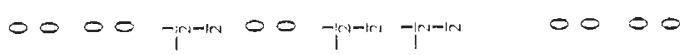

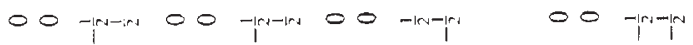

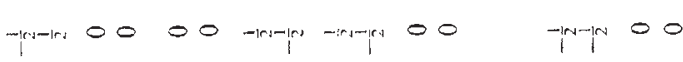

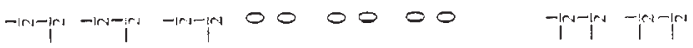

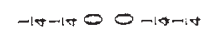

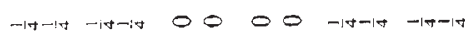

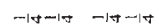

$-40-4-\rightarrow 0-1$

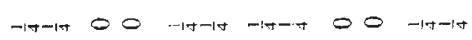

$\operatorname{rin} 00$

$0-\operatorname{lid-14-14-it} 0$

0

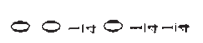

0000

$0-\pi 0-40-14$

00 hithit 00 thith 00 bith

$\operatorname{lin} 00-\sin 0$

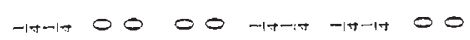

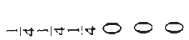

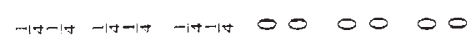

$00000-k$

$000000000000014 \pi$

$0000-10$

00000000 -itm 00

000000 ham 0000

$000-\ln 00$

$00-\ln 000$

0000 Har 000000

$00-1+m 00000000$

$0-10000$

$-i r 00000$

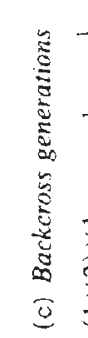

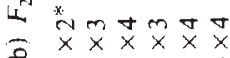

D $x \times x \times x \times$ 


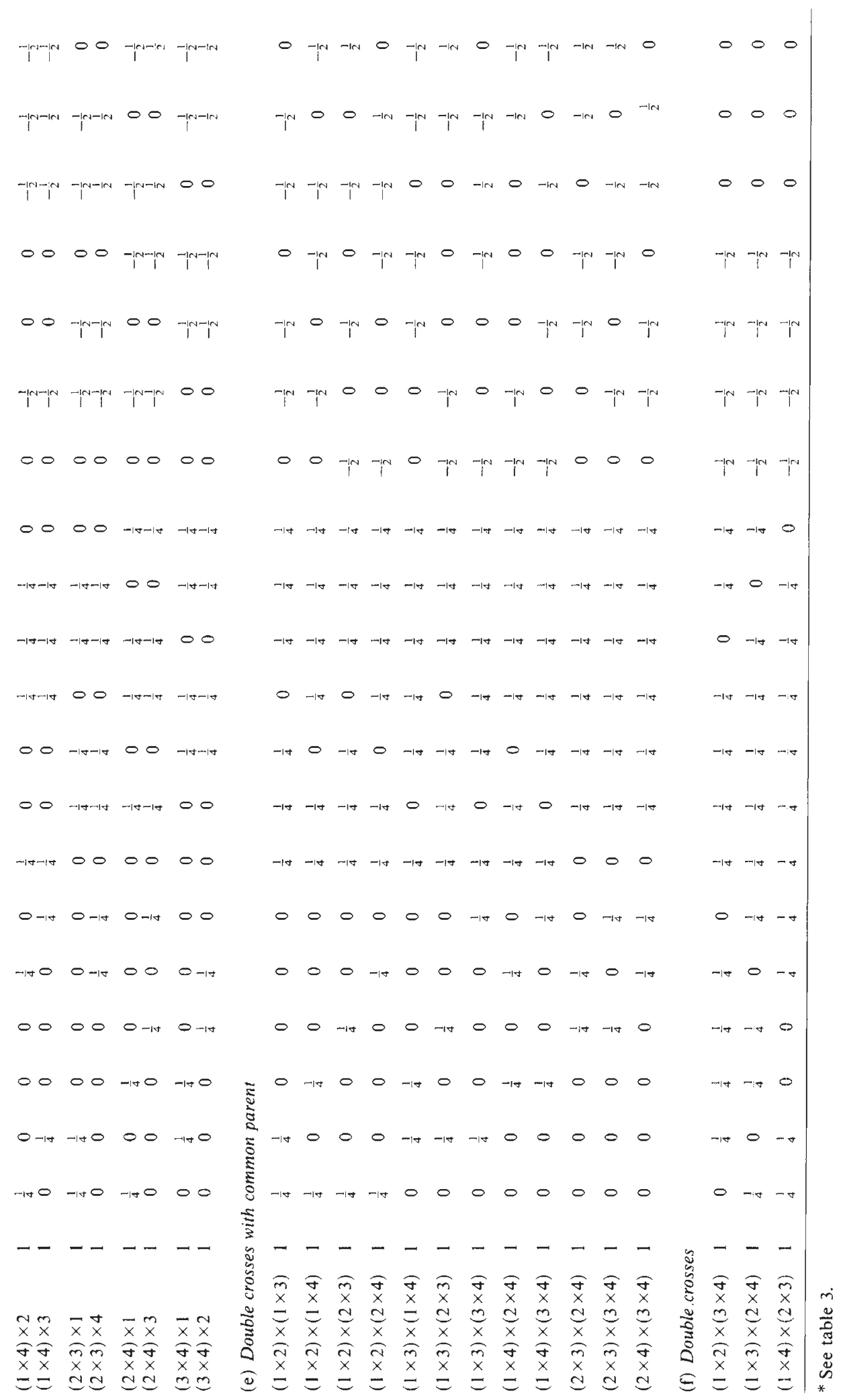


Table 8 Additive genetic variances of random inbreds derived from the $F_{2}$ ss, Backcrosses, three way crosses, three parent double crosses and four parent double crosses and their expectations in terms of additive genetic variances of the six single crosses that are possible amongst four inbred parents

Additive genetic variances of seven groups of loci

Generations

$\begin{array}{lllll}V d_{4} & V d_{5} & V d_{6} & V d_{7} & D_{12}\end{array}$

Additive genetic variances of the six crosses

(a) $F_{2}$ generations

$1 \times 2$
$1 \times 3$
$1 \times 4$
$2 \times 3$
$2 \times 4$
$3 \times 4$

$\begin{array}{lll}1 & 1 & 0 \\ 1 & 0 & 1 \\ 1 & 0 & 0 \\ 0 & 1 & 1 \\ 0 & 1 & 0 \\ 0 & 0 & 1\end{array}$

(b) Backcross generations

\begin{tabular}{|c|c|c|c|}
\hline $\begin{array}{l}(1 \times 2) \times 1 \\
(1 \times 2) \times 2\end{array}$ & $\begin{array}{l}3 \\
4 \\
3 \\
4\end{array}$ & $\begin{array}{l}3 \\
4 \\
3 \\
4\end{array}$ & $\begin{array}{l}0 \\
0\end{array}$ \\
\hline $\begin{array}{l}(1 \times 3) \times 1 \\
(1 \times 3) \times 3\end{array}$ & $\begin{array}{c}\frac{2}{4} \\
3 \\
3\end{array}$ & $\begin{array}{l}0 \\
0\end{array}$ & $\begin{array}{l}3 \\
4 \\
3 \\
4\end{array}$ \\
\hline $\begin{array}{l}(1 \times 4) \times 1 \\
(1 \times 4) \times 4\end{array}$ & $\begin{array}{l}3 \\
\frac{1}{3} \\
4\end{array}$ & $\begin{array}{l}0 \\
0\end{array}$ & $\begin{array}{l}0 \\
0\end{array}$ \\
\hline $\begin{array}{l}(2 \times 3) \times 2 \\
(2 \times 3) \times 3\end{array}$ & $\begin{array}{l}0 \\
0\end{array}$ & $\begin{array}{l}3 \\
3 \\
3 \\
4\end{array}$ & $\begin{array}{l}3 \\
4 \\
3 \\
4\end{array}$ \\
\hline $\begin{array}{l}(2 \times 4) \times 2 \\
(2 \times 4) \times 4\end{array}$ & $\begin{array}{l}0 \\
0\end{array}$ & $\frac{3}{\frac{3}{4}}$ & $\begin{array}{l}0 \\
0\end{array}$ \\
\hline $\begin{array}{l}(3 \times 4) \times 3 \\
(3 \times 4) \times 4\end{array}$ & $\begin{array}{l}0 \\
0\end{array}$ & $\begin{array}{l}0 \\
0\end{array}$ & $\frac{3}{\frac{3}{4}}$ \\
\hline
\end{tabular}

(c) Three way crosses

$(1 \times 2) \times 3$

$(1 \times 2) \times 4$

$(1 \times 3) \times 2$

$(1 \times 3) \times 4$

$(1 \times 4) \times 2$

$(1 \times 4) \times 3$

$(2 \times 3) \times 1$

$(2 \times 3) \times 4$

$(2 \times 4) \times 1$

$(2 \times 4) \times 3$

$\begin{array}{ll}3 & \frac{3}{4} \\ \frac{3}{4} & \frac{3}{4} \\ \frac{3}{4} & \frac{3}{4} \\ 3 & 1 \\ 4 & 0 \\ 3 & 0 \\ 4 & \\ 3 & 1 \\ 1 & 0 \\ 3 & 0 \\ 1 & \frac{3}{4} \\ 1 & \frac{3}{4} \\ 0 & 4 \\ 1 & \frac{3}{4} \\ 0 & \frac{3}{4} \\ 1 & 0 \\ 0 & 1\end{array}$

$\begin{array}{lll}0 & 0 & 1 \\ 0 & 1 & 0 \\ 1 & 1 & 1 \\ 0 & 1 & 1 \\ 1 & 1 & 0 \\ 1 & 0 & 1\end{array}$

$D_{13}$

$\begin{array}{llll}D_{14} & D_{23} & D_{24} & D_{34}\end{array}$

$(3 \times 4) \times 1$

$(3 \times 4) \times 2$

(d) Double crosses with common parent
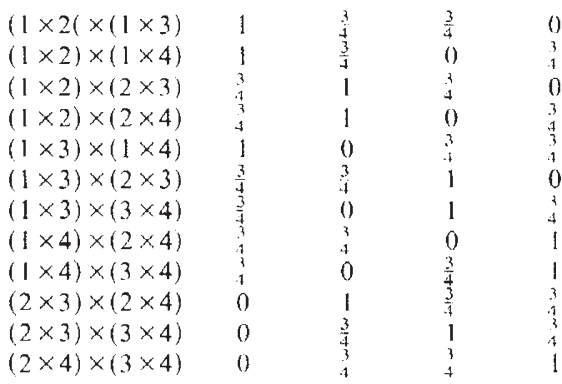

(e) Double crosses

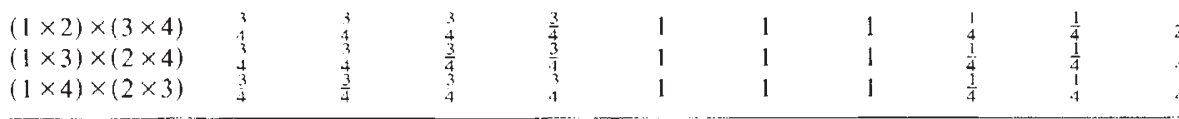

* See table 3 . 
Table 9 Means and variances of the parental, $F_{1}, F_{2}, B_{1}, B_{2}$, three way gross and double cross generations produced by pairwise crossing of varieties $V_{1}, V_{5}, V_{2}$ and $V_{12}$ and crosses $V_{1} \times V_{5}$ and $V_{2} \times V_{12}$ of Nicotiana rustica for the characters flowering time and final height

\begin{tabular}{|c|c|c|c|c|c|c|c|c|c|c|c|}
\hline \multirow[b]{3}{*}{ Generation } & \multicolumn{5}{|c|}{$V_{1} \times V_{5}$ Cross } & \multicolumn{6}{|c|}{$V_{2} \times V_{12}$ Cross } \\
\hline & \multirow[b]{2}{*}{$n$} & \multicolumn{2}{|c|}{ Flowering time } & \multicolumn{2}{|c|}{ Final height } & \multirow[b]{2}{*}{ Generation } & \multirow[b]{2}{*}{$n$} & \multicolumn{2}{|c|}{ Flowering time } & \multicolumn{2}{|c|}{ Final height } \\
\hline & & mean & var. & mean & var. & & & mean & var. & mean & var. \\
\hline$V_{1}$ & 20 & $24 \cdot 80$ & $11 \cdot 33$ & $116 \cdot 00$ & $28 \cdot 95$ & $V_{2}$ & 20 & $43 \cdot 20$ & $8 \cdot 38$ & 90.95 & $64 \cdot 05$ \\
\hline$V_{5}$ & 20 & $24 \cdot 90$ & $6 \cdot 94$ & $120 \cdot 45$ & $172 \cdot 79$ & $V_{12}$ & 20 & $29 \cdot 50$ & $8 \cdot 77$ & $141 \cdot 50$ & $91 \cdot 00$ \\
\hline$F_{1}$ & 40 & $26 \cdot 75$ & $14 \cdot 25$ & $140 \cdot 38$ & $91 \cdot 25$ & $F_{1}$ & 40 & $34 \cdot 45$ & 16.94 & $161 \cdot 53$ & $170 \cdot 31$ \\
\hline$F_{2}$ & 25 & $28 \cdot 80$ & $47 \cdot 50$ & $130 \cdot 88$ & $129 \cdot 53$ & $F_{2}$ & 25 & $37 \cdot 32$ & $39 \cdot 23$ & $144 \cdot 28$ & $361 \cdot 34$ \\
\hline$F_{1} \times V_{1}$ & 25 & $25 \cdot 72$ & $21 \cdot 93$ & 130.88 & $108 \cdot 61$ & $F_{1} \times V_{2}$ & 25 & $41 \cdot 32$ & $17 \cdot 89$ & $141 \cdot 12$ & $476 \cdot 88$ \\
\hline$F_{1} \times V_{5}$ & 25 & $28 \cdot 76$ & $30 \cdot 27$ & 135.96 & $212 \cdot 54$ & $F_{1} \times V_{12}^{2}$ & 25 & $34 \cdot 76$ & $12 \cdot 61$ & $154 \cdot 60$ & $164 \cdot 67$ \\
\hline$F_{1} \times V_{2}$ & 25 & $30 \cdot 08$ & $45 \cdot 58$ & 135.64 & $250 \cdot 64$ & $F_{\mathrm{I}} \times V_{1}$ & 25 & $31 \cdot 60$ & $29 \cdot 33$ & $142 \cdot 88$ & 344.69 \\
\hline$F_{1} \times V_{12}$ & 25 & $29 \cdot 64$ & $40 \cdot 74$ & $150 \cdot 16$ & $271 \cdot 82$ & $F_{1} \times V_{5}$ & 25 & $32 \cdot 64$ & $43 \cdot 07$ & $143 \cdot 84$ & $225 \cdot 06$ \\
\hline $\begin{array}{l}F_{1}(1 \times 5) \times \\
F_{1}(2 \times 12)\end{array}$ & 25 & $31 \cdot 56$ & $85 \cdot 01$ & $142 \cdot 96$ & $385 \cdot 62$ & $\begin{array}{l}F_{1}(2 \times 12) \times \\
F_{1}(1 \times 5)\end{array}$ & 25 & $31 \cdot 60$ & $64 \cdot 42$ & $146 \cdot 16$ & 291.97 \\
\hline
\end{tabular}

Table 10 Weighted least squares estimates of components of means and additive genetic variance $D$ obtained from the basic generations of $V_{1} \times V_{5}$ and $V_{2} \times V_{12}$ crosses for flowering time and final height

Parameters $\overbrace{V_{1} \times V_{5} \text { cross }}^{\text {Flowering time }} \overbrace{V_{2} \times V_{12} \text { cross }}^{\text {Final height }} \overbrace{V_{1} \times V_{5} \text { cross }}^{V_{2} \times V_{12} \text { cross }}$

(a) Components of means

$\begin{array}{lccrr}m & 24 \cdot 86 \pm 0 \cdot 46 & 36 \cdot 35 \pm 0 \cdot 46 & 119 \cdot 48 \pm 1 \cdot 40 & 116 \cdot 23 \pm 1 \cdot 39 \\ {[d]} & - & 6 \cdot 80 \pm 0 \cdot 43 & 3 \cdot 12 \pm 1 \cdot 41 & 25 \cdot 28 \pm 1 \cdot 39 \\ {[h]} & 8 \cdot 17 \pm 2 \cdot 96 & 8 \cdot 27 \pm 2 \cdot 52 & 22 \cdot 03 \pm 2 \cdot 14 & 78 \cdot 14 \pm 9 \cdot 36 \\ {[i]} & - & - & - & - \\ {[j]} & -7 \cdot 35 \pm 2 \cdot 86 & - & - & -22 \cdot 43 \pm 9 \cdot 45 \\ {[l]} & -6 \cdot 28 \pm 2 \cdot 96 & -10 \cdot 17 \pm 2 \cdot 56 & - & -32 \cdot 83 \pm 9 \cdot 53 \\ \chi_{(n)}^{2} & 1 \cdot 32_{(2)}^{\mathrm{NS}} & 0 \cdot 35_{(2)}^{\mathrm{NS}} & 3 \cdot 88_{(3)}^{\mathrm{NS}} & 0 \cdot 53_{(1)}^{\mathrm{NS}}\end{array}$

(b) Additive genetic variance

$\begin{array}{lllll}D & 65 \cdot 02 & 33.96 & 132 \cdot 96 & 624.45\end{array}$

height $(\mathrm{cm})$ were scored on individual plants and the data were subjected to the conventional statistical and weighted least squares analyses. The means and variances of the generations are given in table 9 and the least squares estimates of the genetic parameters of means and variances tabulated in table 10. The estimates of the parameters were obtained from the basic generations of the two crosses.

We shall use estimates of $m,[d]$ and $D$ to obtain abscissa values for predicting the properties of random inbred lines that can be extracted from the $F_{2}, B_{1}$ and $B_{2}$ generations of each cross. For each character we obtain these values by calculating $(S t-m) / \sqrt{D}, \quad\left(S t-m-\frac{1}{2}[d]\right) / \sqrt{\frac{3}{4} D}$ and $\left(S t-m+\frac{1}{2}[d]\right) / \sqrt{\frac{3}{4} D}$, respectively, where the standard $S t$ takes two alternative scores of the largest and the smallest inbred amongst the forty $(20 \mathrm{~B}$ and $20 \mathrm{D})$ inbred families. The predicted proportions of random inbred lines that are expected to score smaller than $\bar{P}_{\text {smallest }}$ or greater than $\bar{P}_{\text {largest }}$ are then obtained by solving the relevant normal probability integrals (Jinks and Pooni, 1976). These are tabulated in table 11.

Table 11 Predicted proportions of random inbred lines extractable from the $F_{2}$ and the first backcross generations of the $V_{1} \times V_{5}$ and $V_{2} \times V_{12}$ crosses of Nicotiana rustica

\begin{tabular}{|c|c|c|c|c|}
\hline \multirow[b]{2}{*}{ Generation } & \multicolumn{2}{|c|}{ Flowering time } & \multicolumn{2}{|c|}{ Final height } \\
\hline & $\geqq \bar{P}_{\text {largest }}$ & $\leqq \bar{P}_{\text {smallest }}$ & $\geqq \bar{P}_{\text {largest }}$ & $\leqq \bar{P}_{\text {smallest }}$ \\
\hline$F_{3}(1 \times 5) \times V_{1}$ & 0.0001 & $0 \cdot 147$ & 0.000 & 0.000 \\
\hline$F_{1}(1 \times 5) \times V_{5}$ & 0.0001 & $0 \cdot 147$ & 0.000 & 0.000 \\
\hline$F_{2}(1 \times 5)$ & $0 \cdot 003$ & $0 \cdot 184$ & 0.001 & $0 \cdot 000$ \\
\hline$F_{1}(2 \times 12) \times V_{2}$ & $0 \cdot 085$ & 0.000 & 0.007 & 0.049 \\
\hline$F_{1}(2 \times 12) \times V_{12}$ & 0.003 & 0.001 & 0.099 & 0.002 \\
\hline$F_{2}(2 \times 12)$ & $0 \cdot 038$ & 0.001 & 0.053 & 0.027 \\
\hline
\end{tabular}


The present experiment also allows us to predict the properties of second cycle hybrids which can be produced by (a) randomly mating $B$ inbreds, (b) randomly mating $D$ inbreds, (c) intermating $B$ and $D$ inbreds and (d) the crossing of $B$ and $D$ inbreds with the four parents, $V_{1}, V_{5}, V_{2}$ and $V_{12}$. We estimate the overall means $\overline{S C H}$ and genetic variances $\left(\sigma_{b S C H}^{2}\right)$ of these populations from the $F_{2}$, double cross, three way cross or backcross generations (as described in section 6 ) and make predictions using the formulae devised by Toledo et al. (1984a, $b)$ and Pooni et al. (1985). Again, we use the extreme inbred lines as standard genotypes and obtain for each population the proportions of second cycle hybrids that will score $\leqq \bar{P}_{\text {smallest }}$ or $\geqq \bar{P}_{\text {largest }}$ by integrating the areas under a standard normal curve from $-\infty$ to $\left(\bar{P}_{\text {smallest }}-\overline{S C H}\right) / \sqrt{\sigma_{b S C H}^{2}}$ and from $\left(\bar{P}_{\text {largest }}\right.$ $\overline{S C H}) / \sqrt{\sigma_{b S C H}^{2}}$ to $+\infty$. These proportions are given for flowering time and final height in table 12.

\section{7ii. Observed results}

Data recorded on $20 \mathrm{~B}$ and $20 \mathrm{D}$ inbred lines and four triple test crosses produced by crossing each set of inbred lines to the $V_{1}, V_{5}$ and $F_{1}\left(V_{1} \times V_{s}\right)$ and $V_{2}, V_{12}$ and $F_{1}\left(V_{2} \times V_{12}\right)$ testers provide the observed proportions for each of the 11 second cycle hybrid populations specified in table 12 . The experiment was conducted during the summer of 1978 when 10 plants for each of the $280(20 \times 2 \times 7)$ families were raised in a single block with individual plant randomisation. Average scores of
20 families in each set of $F_{\infty} \times$ variety crosses were used directly to obtain the genotypic proportions of hybrids corresponding with $\geqq \bar{P}_{\text {largest }}$ and $\leqq \bar{P}_{\text {smallest }}$. Data from

$$
\begin{aligned}
& F_{\infty}\left(V_{1} \times V_{5}\right) \times F_{1}\left(V_{1} \times V_{5}\right), \\
& F_{\infty}\left(V_{1} \times V_{5}\right) \times F_{1}\left(V_{2} \times V_{12}\right), \\
& F_{\infty}\left(V_{2} \times V_{12}\right) \times F_{1}\left(V_{2} \times V_{12}\right)
\end{aligned}
$$

and

$$
F_{\infty}\left(V_{2} \times V_{12}\right) \times F_{1}\left(V_{1} \times V_{5}\right)
$$

sets of crosses however could only be used to obtain phenotypic proportions because in each case individual plants were the sole representatives of second cycle hybrids. The standards used as $P_{\text {largest }}$ and $P_{\text {smallest }}$ were the same $\left(D_{10}\right.$ and $\left.D_{17}\right)$

\begin{tabular}{|c|c|c|c|c|}
\hline & \multicolumn{2}{|c|}{ Flowering time } & \multicolumn{2}{|c|}{ Final height } \\
\hline & 1983 & 1978 & 1983 & 1978 \\
\hline & $43 \cdot 10$ & $54 \cdot 78$ & $150 \cdot 30$ & \\
\hline$D 18$ & $25 \cdot 70$ & $24 \cdot 80$ & $97 \cdot 40$ & \\
\hline
\end{tabular}
for both the prediction (1983) and observation (1978) experiments and their scores were as follows:

The observed proportions are tabulated alongside predictions in table 12 .

\section{CONCLUSIONS}

\begin{tabular}{|c|c|c|c|c|c|c|c|c|}
\hline \multirow{3}{*}{$\begin{array}{l}\text { Second cycle } \\
\text { hybrids }\end{array}$} & \multicolumn{4}{|c|}{ Flowering time } & \multicolumn{4}{|c|}{ Final height } \\
\hline & \multicolumn{2}{|c|}{$\geq \bar{P}_{\text {largest }}$} & \multicolumn{2}{|c|}{$\leqq \bar{P}_{\text {smaliest }}$} & \multicolumn{2}{|c|}{$\geqq \bar{P}_{\text {lisrgest }}$} & \multicolumn{2}{|c|}{$\leq \bar{P}_{\text {smallest }}$} \\
\hline & Predicted & Observed* & Predicted & Observed & Predicted & Observed & Predicted & Observed \\
\hline$B$ inbreds $\times V_{1}$ & 0.000 & $0 \cdot 000$ & 0.003 & $0.050 t$ & 0.000 & 0.000 & 0.000 & 0.000 \\
\hline$B$ inbieds $\times V_{5}$ & 0.000 & 0.000 & $0 \cdot 055$ & $0 \cdot 000$ & 0.010 & 0.000 & 0.000 & 0.000 \\
\hline$D$ inbreds $\times V_{2}$ & 0.013 & $0 \cdot 000$ & $0 \cdot 000$ & 0.000 & 0.206 & $0 \cdot 250$ & $0 \cdot 000$ & $0 \cdot 000$ \\
\hline$D$ inbreds $\times V_{12}$ & 0.000 & $0 \cdot 000$ & $0 \cdot 000$ & 0.000 & 0.356 & 0.000 & 0.000 & $0 \cdot 000$ \\
\hline$B$ inbreds $\times V_{2}$ & 0.001 & 0.000 & 0.006 & 0.000 & 0.027 & 0.000 & 0.000 & 0.000 \\
\hline$B$ inbreds $\times V_{12}^{2}$ & 0.000 & 0.000 & 0.006 & 0.050 & 0.115 & 0.000 & 0.000 & 0.000 \\
\hline$D$ inbreds $\times V_{1}$ & $0 \cdot 000$ & $0 \cdot 000$ & 0.000 & 0.000 & 0.147 & $0 \cdot 000$ & $0 \cdot 000$ & 0.000 \\
\hline$D$ inbreds $\times V_{s}$ & 0.001 & $0 \cdot 000$ & 0.001 & 0.000 & 0.003 & 0.000 & $0 \cdot 000$ & 0.000 \\
\hline$B$ inbreds $\times B$ inbreds & 0.001 & 0.000 & 0.030 & 0.131 & 0.000 & 0.025 & 0.000 & 0.005 \\
\hline$D$ inbreds $\times D$ inbreds & 0.034 & 0.015 & 0.000 & 0.067 & $(0 \cdot 209$ & $0 \cdot 151$ & 0.000 & 0.000 \\
\hline$B$ inbreds $\times D$ inbreds & 0.021 & 0.003 & 0.029 & 0.081 & 0.161 & 0.094 & 0.000 & 0.000 \\
\hline
\end{tabular}

In the preceding sections we have shown that the standard models of biometrical genetics can be

Table 12 Predicted and observed proportions of second cycle hybrids with scores $\geqq \vec{P}_{\text {largest }}$ or $\leqq \bar{P}_{\text {smaliest }}$ for flowering time and final height

* Predicted and observed are obtained from 1983 and 1978 experiments, respectively.

$\dagger$ Observed proportions are genotypic for all except $B \times B, D \times D$ and $B \times D$ populations. 
made applicable to the means and variances of the 55 generations which can be produced by crossing four pure breeding varieties and their six $F_{1}$ 's in all possible combinations. With two alleles per locus the expectations of the means and additive genetic component of the variances for all 55 generations can be written in terms of parameters appropriate for a cross between a single pair of varieties (tables 3, 4 and 5). Additional parameters are required, however, for the dominance component of the variances and for the additive genetic-dominance covariance (table 7). The consequences of having three or four alleles per locus can be accommodated only by confounding their effects with existing parameters in the models ( $\mathrm{sec}$ tion 3ii).

The expected means of the populations of pure breeding lines that are extractable from any cross involving up to four parental varieties can be predicted from estimates of the appropriate components of the means obtained from the basic generations of the six single crosses (section 5 and tables 4 and 5). These provide unbiased estimates and predictions even in the presence of non-allelic interactions if these are explicitly allowed for in the expectations.

The expected variances of the populations of pure breeding lines are inflated in the presence of non-allelic interactions and may be inflated or deflated by a linkage dis-equilibrium depending on whether the predominant phase is coupling or repulsion (Jinks and Pooni, 1976; 1981; 1984). Their estimates obtained from the variances of 55 generations are also inflated by non-allelic interactions but not to the same extent and the effect of any linkage dis-equilibrium is greater. Experience has shown, however, that for single crosses the estimates from the early generations are close approximations to the observed variances of the populations of pure breeding lines extractable from them (Jinks and Pooni, 1976; 1981; Pooni and Jinks, 1978; Pooni, Jinks and Jayasekara, 1978). Since the additive genetical parameters of the single and multiple crosses are the same (tables 9 and 10) we anticipate no problems in predicting the variances of the populations of pure breeding lines extractable from multiple crosses from the variances of early generations in the presence of non-allelic interactions and a linkage disequilibrium.

The means and variances of the 55 early generations also provide estimates of the genetical parameters required to predict the properties of populations of second cycle hybrids (section 6). This allows the procedures for predicting the properties of the second cycle hybrids derivable from the $F_{2}$ of a single cross (Toledo, Pooni and Jinks, $1984 a, b$; Pooni, Jinks and Toledo, 1985) to be extended to those produced by (a) randomly crossing the pure breeding lines extractable from any generation of a single cross; (b) pairwise crossing of two sets of pure breeding lines each extracted from a different generation of the same or a different cross and (c) backcrossing of the pure breeding lines to any one of the four parental varieties. By combining these predictions with those for pure breeding lines we can discriminate between crosses and generations within crosses on the basis of their relative potentials for producing superior recombinant inbreds and second cycle hybrids and we can formulate the best breeding strategy to harness the potential and to choose the best end product.

A full experimental test of the theory must await the completion of current field experiments. In the meantime we can draw preliminary conclusions from the results in table 11 . Some of these are very familiar. For example, the $V_{2} \times V_{12}$ cross has a higher potential for transgressive segregation leading to superior recombinant inbred lines than the $V_{1} \times V_{5}$ cross. Thus if we inbreed the $F_{2}$ or either of the backcross generations $\left(B_{1}\right.$ or $\left.B_{2}\right)$ of the $V_{2} \times V_{12}$ cross we have a reasonable probability of producing recombinant inbreds that outperform $\bar{P}_{\text {largest }}$ and $\bar{P}_{\text {smallest }}$ both for final height and flowering time. A large proportion of the recombinant inbreds extractable from the $V_{1} \times V_{5}$ cross will, however, flower earlier than $\bar{P}_{\text {smallest }}$ but hardly any will transgress for final height or late flowering.

For the $V_{1} \times V_{5}$ cross the $F_{2}$ is unconditionally superior to the backcrosses as a source of transgressive inbred segregants. For the $V_{2} \times V_{12}$ cross, however, the backcrosses are expected to produce a somewhat higher frequency of transgressive inbred segregants than the $F_{2}$ but in the direction of the backcross parent only and at the expense of a corresponding reduction in frequency in the other direction (table 11). Because 75 per cent of the genes in the inbred population derived from a first backcross come from one parent a high proportion of these inbreds are expected to cluster around the mean of this parent. For example, if allelic differences of equal effect are segregating at $k$ loci a proportion $(k ! / r !(k-r) !)\left(3^{k-r} / 4^{k}\right)$ of the initial lines derivable from a first backcross will have increasing alleles at $r$ more or $r$ less of the $k$ loci than the backcross parent, where $r=0$, $1,2 \ldots$ to $k$. This proportion has a maximum when $r=0$, that is when the inbred line has the same phenotype as the backcross parent and it falls off 
rapidly as $r$ increases. It is, however, always greater than $(k ! / r !(k-r) !)\left(\frac{1}{2}\right)^{k}$, the corresponding proportion of inbred lines derivable from the $F_{2}$ of the same cross, when $r \leqq 0.37 k$ but this situation is rapidly reversed when $r \geqq 0 \cdot 37 k$. Hence backcrosses are expected to generate fewer extreme recombinant inbred lines than an $F_{2}$ unless, of course, almost all of the increasing alleles are present in $P_{1}$ and almost all of the decreasing alleles in $P_{2}$ so that a low value of $r$ would produce $P_{\text {largest }}$ and $P_{\text {smallest }}$.

The predicted and observed proportions of second cycle hybrids obtained from the various sources that meet the criteria ( $\geqq \bar{P}_{\text {largest }}$ or $\leqq \bar{P}_{\text {smallest }}$ ) show a reasonable degree of concurrence bearing in mind that only small samples are available in these preliminary experiments for some of the comparisons (table 12). The predicted proportions with one exception do not differ from those for the inbred lines in table 11 suggesting that the second cycle hybrids that can be produced from any of the generations derivable from crosses of varieties one, two, five and twelve can always be matched in performance by inbred lines extracted from the same source. The exception is final height where a higher proportion of second cycle hybrids are expected to outperform $\bar{P}_{\text {largest }}$ than of inbred lines.

It is particularly noticeable (table 12) that the double cross is inferior to the better of the two single crosses for the proportion of inbred lines, both predicted and observed, that meet the criteria ( $\geqq \bar{P}_{\text {largest }}$ or $\leqq \bar{P}_{\text {smallest }}$ ).

In making the predictions we assume that the genotypic distributions of the first cycle inbreds and second cycle hybrids are approximately normal. This will be true, however, only when all known sources of non-normality, namely, unequal gene frequency, dominance, epistasis, linkage disequilibrium and genotype $\times$ environmental interactions are either absent or the non-normalities they produce cancel each other out (Jinks and Pooni, 1976; Pooni, Jinks and Cornish, 1977; Jinks, 1983; Toledo, Pooni and Jinks, 1984a, $b$; Pooni, Jinks and Toledo, 1985). In general, therefore, we expect some non-normality in the distributions of inbreds and hybrids. Previous experience, however, suggests that the levels of epistasis, linkage disequilibrium and genotype $\times$ environmental interactions encountered in practice cause few difficulties and can be accommodated by the general robustness of the prediction procedures. Directional dominance and unequal gene frequencies, particularly in the backcross populations, however, are more serious sources of non-normality. But even these are not expected to cause serious difficulties unless the number of segregating loci $(k)$ is small and the dominance ratio and the degree of gene association in the parents is high.

Finally, the design of experiment we have proposed for predicting the properties of first cycle inbreds and second cycle hybrids takes the shortest possible time of three seasons, i.e., two seasons for producing seed and a third for conducting the experiment. Further, the generations included in this design are often produced by the breeders for other purposes such as evaluation and selection. The genetical analysis we suggest merely requires that these materials be evaluated simultaneously in a single experiment. This in turn provides useful information on the inbreeding and outcrossing potentials of the breeding material and helps in selecting a suitable method of breeding in the initial stages of a breeding programme.

\section{REFERENCES}

CoOmBs. D. T. 1980. Biometrical Genetics of Tobacco. Ph.D. Thesis, University of Birmingham.

IBBIRHART, S. A. 1964. Theoretical relations among single, threcway, and double cross hybrids. Biometrics, 20, 522539.

JINKS, J. L. 1983. Biometrical Genetics of Heterosis. In Frankel, R. (ed.) Heterosis. Monographs on Theoretical and Applied Genetics. Springer-Verlag, Berlin, pp. 1-46.

IINKS, J. L.. AND PERKINS, J. M. 1972. Predicting the range of inbred lines. Heredity, 28, 399-403.

JINKS, J. L. ANI POONI, H. S. 1976. Predicting the properties of recombinant inhred lines derived by single seed descent. Heredity, 36, 253-266.

JINKS, J. L. AND POON1, H. S. 1981. Properties of pure breeding lines produced by dihaploidy, single seed descent and pedigree inbreeding. Heredity, 46, 391-395.

IINKS, I. I. ANI) P(ONI, H. S. 1984. Comparison of inbred lines produced hy single seed descent and pedigree inbreeding. Heredity, 53, 299-308.

MATHER, K. AND JINKS, J. L. 1982. Biometrical Genetics, 3rd Edn. Chapman and Hall, London.

PI:RKINS, I. M. ANI) IINKS, I. 1. 1973. The assessment and specificity of environmental and genotype-environmental camponents of variability. Heredity, 30, 111-126.

POONI, H. S. ANI) IINKS, J. 1.. 1978. Predicting the properties of recomhinant inhred lines derived by single seed descent for two or more characters simultaneously. Heredity, 40, 349-361

POONI. H. S. AND JINKS, J. L. 1979. Sources and biases of the predictors of the properties of recombinant inhreds produced by single seed descent. Heredity, 42, 41-48.

P()ONI. H. S. JINKS, I. I. AND CORNISH, M. A. 1977. The causes and consequences of non-normality in predicting the properties of recomhinant inbred lines. Heredity, 38, 329338.

POONI, H. S., JINKS, J. L. AND JAYASEKARA, N. E. M. 1978. An investigation of gene action and genotype $\times$ environment interaction in two crosses of Nicotiana rustica hy triple test cross and inbred line analysis. Heredity, 4l, 83-92. 
POONI, H. S., JINKS, J. L. AND TOLEDO, J. F. F. DE. 1985. Predicting and observing the properties of second cycle hybrids using basic generations and inbred line $\times F_{1}$ crosses. Heredity, 54, 121-129.

TAPSFLL, C. R. AND THOMAS, W. T. B. 1983. Cross prediction studies on spring barley. 2. Estimation of genetical and environmental control of yield and its component characters. Theor. Appl. Genet., 64, 353-358.

THOMAS, W. T. B. AND TAPSF.LL, C. R. 1983. Cross prediction studies on spring barley. 1. Estimation of genetical and environmental control on morphological and maturity characters. Theor. Appl. Genet, 64, 345-352.
TOLEDO, J. F. F. DE, POONI, H. S. AND JINKS, J. L. $1984 a$. Predicting the properties of second cycle hybrids produced by intercrossing random samples of recombinant inbred lines. Heredity, 53, 283-222.

TOLEDO, J. F. F. DE, POONI, H. S. ANI) JINKS, J. L. $1984 b$. Predicting the transgressive potentials of Nicotiana rustica crosses for superior recombinant inbreds and second cycle hybrids. Proceedings of the Fifth Meeting of the Biometrics Section of Eucarpia, Hohenheim, Stuttgart. 151-166. 